\title{
Design Improvements for Proportional Control of Autonomous Wheelchairs Via 3DOF Orientation Tracker
}

\author{
Christian Mandel ${ }^{1}$, Udo Frese ${ }^{2}$, and Thomas Röfer ${ }^{2}$ \\ 1 University of Bremen, P.O.Box 330440, 28334 Bremen, Germany, \\ cmandel@uni-bremen.de, \\ WWW home page: http://wWw.informatik. uni-bremen.de/ ${ }^{\mathrm{cman} /}$ \\ 2 DFKI Lab Bremen, Safe and Secure Cognitive Systems, \\ Robert-Hooke-Straße 5, 28359 Bremen, Germany
}

\begin{abstract}
This paper presents a three degrees of freedom orientation tracker as suitable controlling equipment for an automated wheelchair. Mounted at the back of an operator's head by the help of an easy to wear frontlet, the device permanently outputs the user's head posture which can be used as a joystick-like signal. Within an experimental evaluation we demonstrate the applicability of the proposed control interface even for untrained users.
\end{abstract}

\section{Introduction}

Electrical wheelchairs make up one of the fundamental tools that ease the rehabilitation time or the everyday life of disabled people. In most of the cases the handicapped's inability to walk by his/her own is substituted by a general control loop that embeds the operator's remaining sensomotor capabilities into the vehicle's actuating body. The most common example for these kinds of systems requires the user to push a joystick into the desired direction of movement and therewith triggering a proportional translatory and rotatory motor activity of the wheelchair. Unfortunately, such an intuitive interface is impractical for certain groups of patients. For example, people suffering from certain spinal cord injury dysfunctions can only move body parts that are located above their shoulders. In this work we propose the use of a head-mounted three degrees of freedom orientation tracker ${ }^{3}$ to give the target group mentioned above an easy way of controlling their vehicle by intuitive head movements. In particular we will pursue our own preliminary work[1], that culminated in an empiric study with 15 untrained participants, each of them comparing the applicability of our basic implementation of an IMU-based head-joystick versus a common joystick.

We begin in section 2 with a general survey on methods that focus on human-robot interfaces for automated wheelchairs. In section 3 we continue with

${ }^{3}$ Throughout this work we will abbreviate three degrees of freedom orientation tracker or rather inertial measurement unit by IMU. 
the presentation of the (semi-)autonomous wheelchair Rolland that is used in our laboratory as a demonstrator for developments in the field of service- and rehabilitation-robotics. Confer [2-4] for a broader overview. An entering guide to the major software modules applied throughout this work completes this section. Then in section 4 we introduce the basic implementation of an IMU-based head-joystick that converts information about the pilot's head posture into appropriate control commands for a (semi-)autonomous wheelchair. After section 5 proceeds with several technical improvements that draw on the algorithmic evaluation of the IMU's output data, section 6 presents results of an experimental evaluation that compares the performance of untrained participants testing the different versions of the proposed head-controller. We conclude in section 7 with a critical assessment of the gathered results.

\section{Related Work}

Human-robot interaction (HRI) is an active research field that investigates methods of interfacing with (semi)-autonomous robot systems. In the following we give a short survey on sophisticated techniques that enable paralysed people to operate electrical wheelchairs.

Jaffe proposes in [5] a head position interface that has been evaluated within a clinical trial [6]. Due to the applied sensorial hardware, i.e. two ultrasonic sensors mounted at the wheelchair's headrest, the system can only measure the head's movement within a two-dimensional plane that lies parallel to the ground. Chen and colleagues propose in a more recent work [7] the use of two inertial sensors that are attached to the head of a wheelchair-bound person. The overall system triggers discrete commands, e.g. $70 \mathrm{~cm} / \mathrm{s}$ translational speed when the operator moves his/her head forward once, or $100 \mathrm{~cm} / \mathrm{s}$ translational speed when the operator moves his/her head forward twice. A different approach is presented by Canzler and Kraiss [8]. By the help of computer vision, the authors analyse facial features like head posture, direction of gaze, and lip movement. Hereby they are able to recognize at least four gesture dependent commands like go, stop, left and right. The general computer interface EagleEyes is presented by Gips [9]. The system measures the relative orientation of the user's eyes w.r.t. the current head posture by sensing the electro-oculographic potential via simple electrodes mounted around the eyes. EagleEyes has been integrated into Wheelesley, a robotic wheelchair system [10]. By interpreting the sensorial signals as cursor coordinates, it is shown that handicapped people can control basic driving behaviours by selecting the appropriate buttons on a graphical user interface.

\section{Hardware and Software Prerequisites}

Our experimental platform Rolland is based on the electrical wheelchair Champ 1.594, produced by the German company Meyra. With its modular configuration it is supported by miscellaneous hardware components. For the experiments that where conducted throughout this work, Rolland was supplied by two Siemens 
$L S 4$ laser range finders. Mounted beneath the feet of a human operator, they sense distances to nearby obstacles. The second basic component comes along by two Lenord+Bauer Gel 248 incremental encoders, that measure the rotational speeds of the two actuated wheels for doing dead-reckoning.

For the purpose of measuring globally correct head posture angles we apply the XSens MTx IMU, that is a small-scale ${ }^{4}$ electronical device which provides inertial information, namely 3D acceleration, 3D rate of turn, and 3D earthmagnetic field [11]. By the combination of the output of the device's internal accelerometers, gyroscopes, and magnetometers the IMU outputs also drift-free absolute 3D orientation data. In order to use this device for measuring the posture of a person's head, we have mounted the IMU at the head's back with the help of a small-sized and easy to wear frontlet, cf. Fig.1(a).

The two most important software modules that are involved throughout this work are the Drive Controller and the so-called Safety Layer. The former one gathers raw data coming from the IMU, and converts this information into desired translational and rotational speeds for the vehicle. Confer sections 4 and 5 for a detailed account on the proposed drive controller. The key concept in the implementation of the safety layer is the Virtual Sensor. For a given initial orientation $(\theta)$ of the robot and a pair of translational $(v)$ and rotational $(w)$ speeds, it stores the indices of cells of a local obstacle map that the robot's shape would occupy when initiating an immediate full stop manoeuvre. A set of precomputed virtual sensors for all combinations of $(\theta, v, w)$ then allow us to check the safety of any driving command issued by the drive controller.

\section{Basic Implementation of an IMU-based Head-Joystick}

In this section we present implementation details for a software module that interprets IMU-readings as head-joystick signals. Within the targeted application scenario, the operator permanently controls his vehicle by pitching his head forwards and backwards in order to control translational velocity. In analogy, left and right roll movements of the user's head control rotational velocity. For a clarification of the possible directions of head movement confer Fig. 1(a). Throughout this work we have set the configuration of the IMU to output Euler angles that describe the orientation of the IMU's local coordinate system $S$ with respect to the fixed global coordinate system $G$. We refer to a single IMU reading by the triple $\mathcal{I}=(\psi, \varphi, \theta)$. The nomenclature of the three axis of rotation and the valid output intervals are given in (1).

$$
\begin{aligned}
& \psi=\text { pitch = rotation around } X_{G} \in\left[-90^{\circ} \ldots 90^{\circ}\right] \\
& \varphi=\text { roll }=\text { rotation around } Y_{G} \in\left[-180^{\circ} \ldots 180^{\circ}\right] \\
& \theta=\text { yaw }=\text { rotation around } Z_{G} \in\left[-180^{\circ} \ldots 180^{\circ}\right]
\end{aligned}
$$

\footnotetext{
${ }^{4}$ outline dimensions: $53 * 38 * 21 \mathrm{~mm}^{3}$, weight: $30 \mathrm{~g}$
} 


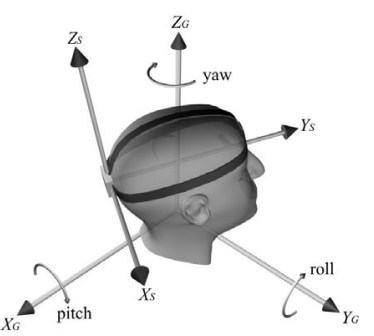

(a) All measured angles describe the posture of the IMU's local coordinate system $S$ w.r.t. a fixed global coordinate system $G$.
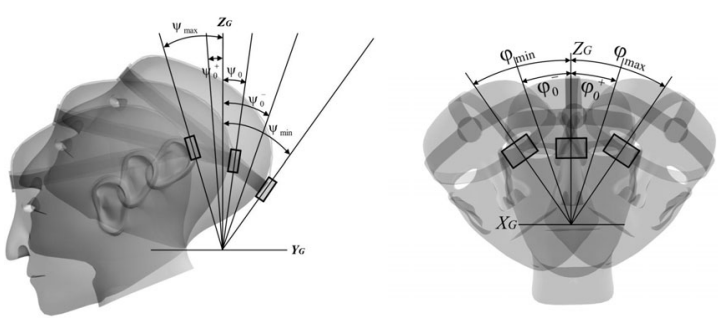

(b) left: Maximal pitch deflection $\psi_{\max }$, minimal pitch deflection $\psi_{\text {min }}$, and mean pitch deflection $\psi_{0} \cdot \psi_{0}^{+}$ and $\psi_{0}^{-}$describe the pitch dead zone, i.e. only pitch angles exceeding these values will be accepted as control commands. right: Roll angles $\varphi_{\max }, \varphi_{0}^{+}, \varphi_{0}, \varphi_{0}^{-}, \varphi_{\min }$ are defined in analogy.

Fig. 1. The IMU that is mounted at the back of the user's head with the help of an easy to wear frontlet outputs head posture angles that are converted into steering commands. The stressed angles in the middle and right illustration are calculated during the calibration phase of the imu and given in the global coordinate system $G$.

\subsection{Head-Joystick Calibration}

Before the IMU can be used as a joystick-replacement, the device has to be calibrated in the sense that the minimal, the mean, and the maximal deflection of the operator's head has to be adopted. For this purpose, let $P_{\min }$ and $P_{\max }$ be two sets of IMU-readings that have been taken while the user has pitched his head with maximal deflection forwards and backwards respectively. In analogy, let $R_{\min }$ and $R_{\max }$ be two sets of IMU-readings that characterise the minimal and the maximal roll deflection of the user's head. By computing the arithmetic mean of the $\psi$ and $\varphi$ components of all four sets, we get a good approximation for the user's minimal and maximal head deflections, i.e. $\psi_{\max }, \psi_{\min }, \varphi_{\max }$, and $\varphi_{\min }$. Furthermore we describe the rest position of the person's head by $\psi_{0}=$ $\frac{\psi_{\max }+\psi_{\min }}{2}$ and $\varphi_{0}=\frac{\varphi_{\max }+\varphi_{\min }}{2}$. In order to allow the wheelchair-bound person to move his or her head somewhat without causing an unintended command, we now define a dead zone around $\psi_{0}$ and $\varphi_{0}$ by introducing $\psi_{0}^{+}, \psi_{0}^{-}, \varphi_{0}^{+}$, and $\varphi_{0}^{-}$, cf. Fig. 1(b) for an illustration of the defined angles. A valid head-joystick command is now solely defined for input values $\left(\psi_{\text {valid }}, \varphi_{\text {valid }}\right)$ that satisfy $(2)$.

$$
\begin{aligned}
& \psi_{\text {valid }} \in\left[\psi_{\max } \ldots \psi_{0}^{+}\right] \cup\left[\psi_{0}^{-} \ldots \psi_{\min }\right] \\
& \varphi_{\text {valid }} \in\left[\varphi_{\max } \ldots \varphi_{0}^{+}\right] \cup\left[\varphi_{0}^{-} \ldots \varphi_{\min }\right]
\end{aligned}
$$

\subsection{Computation of Proportional Control Commands}

After having defined the domain of valid head posture angles, we can specify the transfer function for mapping these angles onto the rotational and translational speeds of the vehicle. Initially we chose a linear transfer function in analogy to 
the proportional characteristic curve of a common joystick. Constants $c_{v}$ and $c_{w}$ in the formulae for the computation of translational and rotational speeds (3) are used to map $v$ and $w$ onto the velocity-domain of a particular vehicle.

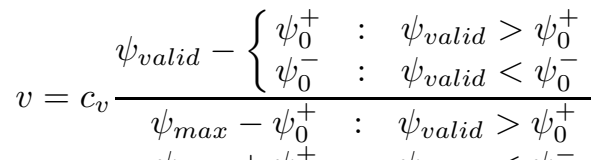

$$
\begin{aligned}
& -\psi_{\min }+\psi_{0}^{+}: \psi_{\text {valid }}<\psi_{0}^{-}
\end{aligned}
$$

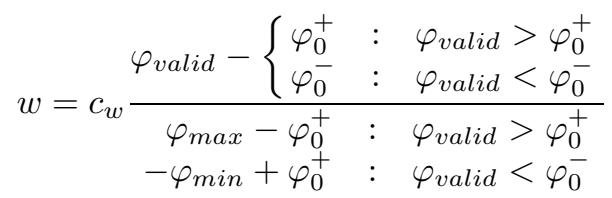

For an illustration of the resulting linear transfer function confer Fig.2(a). Please note that the figure also depicts a quadratic and a cubic transfer function that will be discussed in section 5.2 .

\section{Design-Improvements for an IMU-based Head-Joystick}

At the end of a first implementation phase we conducted an empirical study in which 15 participants tested a version of an IMU-based head-joystick that corresponds to the described system in section 4 . This section now introduces several improvements relating to the algorithmic treatment of the IMU's head posture measurements resulting in an increased overall driving performance.

\subsection{Dynamic Dead Zone for Head Roll Movements}

A major shortcoming in the first implementation of an IMU-based head-joystick could be observed in situations of unrestricted straight ahead movement. While moving with high translational speed on an almost straight line, roll movements of the user's head that slightly exceeded the static bounds of the $\varphi$ dead zone caused significant oscillations around the desired path. This effect can easily be identified by the comparison of Fig. 3(a) and Fig. 3(b). The upper part of both plots depict paths that have been executed in a straight corridor. In order to overcome the described problem, we have implemented a dynamic dead zone for the head's roll movements. The basic idea is to increase the clearance of unconsidered roll movements for driving situations with high translational speed, i.e. for situations where the user's head is far pitched up or down respectively. A reformulation of the roll dead zone is given in (4) and pictured in Fig.2(b).

$$
\begin{array}{r}
\varphi_{\text {valid }} \in\left[\varphi_{\max } \ldots \dot{\varphi}_{0}^{+}\right] \cup\left[\dot{\varphi}_{0}^{-} \ldots \varphi_{\min }\right] \\
\text { with } t=c_{d z} \frac{|\psi|-\psi_{0}^{+}}{\psi_{\max }-\psi_{0}^{+}}\left(\varphi_{\max }-\varphi_{0}^{+}\right), \\
\dot{\varphi}_{0}^{+}=\varphi_{0}^{+}+t, \\
\dot{\varphi}_{0}^{-}=\varphi_{0}^{-}-t
\end{array}
$$




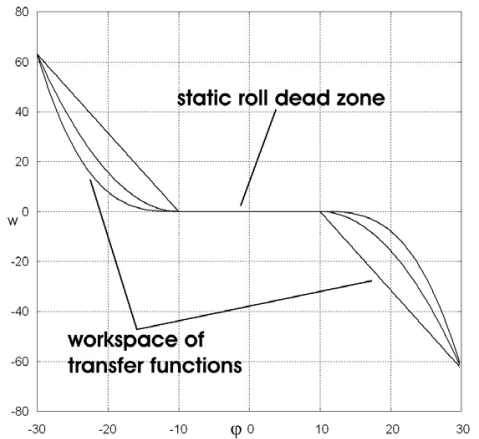

(a) Linear, quadratic, and cubic transfer functions that map the head's roll angle $\varphi$ onto the rotational velocity $w$. The $\varphi$ dead zone ranges from $-10^{\circ}$ to $10^{\circ}$.

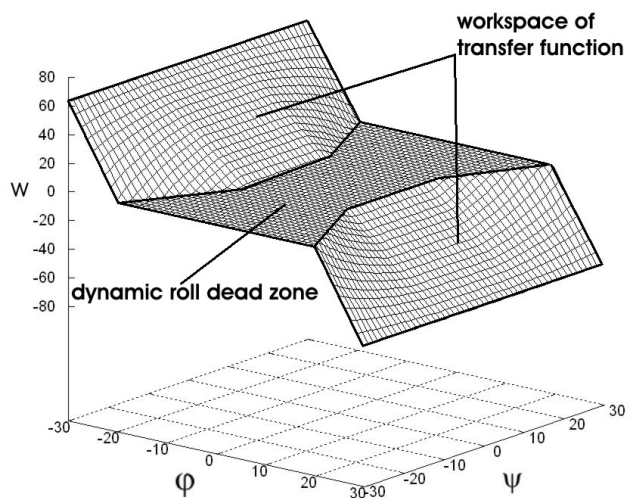

(b) Linear transfer function that maps the head's roll angle $\varphi$ and its pitch angle $\psi$ onto the rotational velocity $w$. We call this two-valued function a dynamic roll dead zone because it also constrains the validity of a given roll angle to the current pitch angle, that is actually deciding the translational speed $v$.

Fig. 2. Both plots show transfer functions for head roll movements, i.e. the functional dependency of the rotational velocity $w$ from the head's roll angle $\varphi$ in the left figure, and the dependency of $w$ from the head's roll angle $\varphi$ and its pitch angle $\psi$ respectively.

\subsection{Transfer Functions of Higher Order}

Unintended slight head movements that exceed the pitch or roll dead zone, cause undesirable translational or rotational movements. Even if the formulation of a dynamic dead zone for the head's roll angle scales down this effect, there persists the basic necessity to reduce oscillations in the driven path. For this reason we have implemented transfer functions of higher order that weight, in contrast to a common proportional joystick, input angles by a quadratic or cubic transfer function respectively. Fig. 2(a) exemplarily shows a linear, a quadratic, and a cubic transfer function for the head's roll angle $\varphi$. It is easy to see that head movements throughout the whole workspace are weaklier assessed by higher order transfer functions than by linear ones.

\section{Experimental Evaluation}

The refined version of an IMU-based head-joystick has been tested in a further experimental evaluation phase. In analogy to the previous survey, we asked 15 participants to steer Rolland on an approximately $25 \mathrm{~m}$ long s-shaped course in our laboratory. In particular we studied the test person's ability to hold the vehicle on a straight line course without causing intense oscillations. A first look 


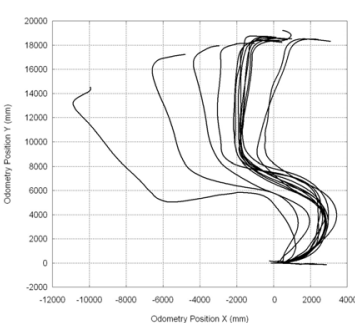

(a) Paths driven by a standard joystick.

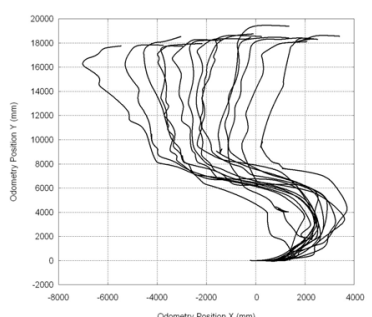

(b) Paths driven by the ba- (c) sic version of a headjoystick, cf. section 4 .

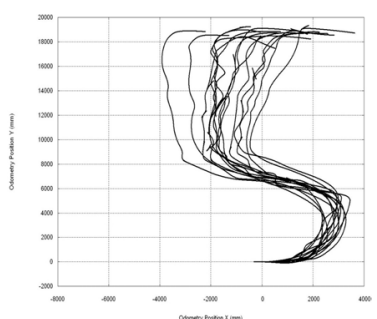

(c) Paths driven by the refined version of a headjoystick, cf. section 5 .

Fig. 3. In each of the two evaluation phases we asked 15 test persons to navigate Rolland on an approximately $25 \mathrm{~m}$ long s-like shape. All three plots show the driven paths in drifting odometry coordinates, whereby we can explain the strong deviations culminating in the aimed target at the upper right part of the plots.

on Fig. 3 reveals the paths that where driven by a common joystick and by the head-joystick in its two different stages of development. Although we expected the improved head-joystick version in Fig. 3(c) to show less variations from accurate straight ahead movement, minor problems in precise rotational control were still observable. A different point of view is given in Table 1. Compared with the basic implementation of the head-joystick, the refined version that applied a quadratic transfer function along with a dynamic roll dead zone, outperforms the basic version in terms of safety layer interventions. This metric predicates the driver's ability to safely manoeuvre along the given course. Confer section 3 for a brief account on the safety layer's operation mode.

\section{Conclusion}

For a special class of patients relying on electrical wheelchairs that are controllable without hand-use, we have implemented an interface based on a 3dof orientation tracker that is mounted at the back of the operator's head. We have shown that the evaluation of the user's head-posture is appropriate for controlling translational and rotational velocities of an automated wheelchair. Although the conducted experiments support this appreciation, their analysis leave several open questions. For example it remains unclear how actually handicapped people judge the proposed user interface. Therefore we have to conduct further long time experiments with the targeted audience. A second open question is whether problems in the rotational control can be solved by more sophisticated filtering techniques that are applied to the sensor's raw data. Finally it is worth to consider the user friendliness of a device that is attached to the back of the head and currently connected via a serial data cable. A final version for example should exchange the measured data with the computing unit via a wireless link. 
Table 1. The table contrasts benchmark data of 15 participants that tested the autonomous wheelchair Rolland by using a common joystick, a basic implementation of an IMU-based head-joystick, and a refined version of the head-joystick. For a discussion of the recorded data confer section 6 .

\begin{tabular}{|lccc|}
\hline Criterion & $\begin{array}{c}\text { Common } \\
\text { Joystick }\end{array}$ & $\begin{array}{c}\text { IMU as } \\
\text { Head-Joystick }\end{array}$ & $\begin{array}{c}\text { Refined } \\
\text { Head-Joystick }\end{array}$ \\
\hline$\phi$ time of travel $[\mathrm{s}]$ & 30.73 & 55.03 & 61.78 \\
$\phi$ length of travel $[\mathrm{m}]$ & 22.45 & 25.03 & 26.88 \\
$\phi$ average speed $[\mathrm{m} / \mathrm{s}]$ & 0.76 & 0.50 & 0.49 \\
$\phi$ safety layer $[\mathrm{ms}]$ & 111.04 & 445.76 & 93.87 \\
$\quad$ interventions & & & \\
\hline
\end{tabular}

\section{ACKNOWLEDGMENTS}

This work has been partly funded by the Deutsche Forschungsgemeinschaft (DFG) in context of the Sonderforschungsbereich/Transregio 8 Spatial Cognition and by the EU project SHARE-it (FP6-045088).

\section{References}

1. Mandel, C., Röfer, T., Frese, U.: Applying a 3dof orientation tracker as a humanrobot interface for autonomous wheelchairs. In: Proceedings of the IEEE Intl. Conf. on Rehabilitation Robotics (ICORR). (2007) Submitted.

2. Lankenau, A., Röfer, T.: A safe and versatile mobility assistant. IEEE Robotics and Automation Magazine 8(1) (2001) 29-37

3. Mandel, C., Hübner, K., Vierhuff, T.: Towards an autonomous wheelchair: Cognitive aspects in service robotics. In: Proceedings of Towards Autonomous Robotic Systems (TAROS). (2005)

4. Mandel, C., Frese, U., Röfer, T.: Robot navigation based on the mapping of coarse qualitative route descriptions to route graphs. In: Proceedings of the IEEE Intl. Conf. on Intelligent Robots and Systems (IROS). (2006)

5. Jaffe, D.L.: An ultrasonic head position interface for wheelchair control. Journal of Medical Systems 6(4) (1982) 337-342

6. Ford, J.M.: Ultrasonic head controller for powered wheelchairs. Journal of Rehabilitation Research and Development 32(3) (1995) 280-284

7. Chen, Y.L., Chen, S.C., Chen, W.L., Lin, J.F.: A head orientated wheelchair for people with disabilities. Disability and Rehabilitation 25(6) (2003) 249-253

8. Canzler, U., Kraiss, K.F.: Person-adaptive facial feature analysis for an advanced wheelchair user-interface. In: Proceedings of the IEEE Intl. Conf. on Mechatronics and Robotics. (2004)

9. Gips, J.: On building intelligence into eagleeyes. In: Lecture Notes in AI: Assistive Technology and Artificial Intelligence. (1998)

10. Yanco, H.: Wheelesley, a robotic wheelchair system: Indoor navigation and user interface. In: Lecture Notes in AI: Assistive Technology and Artificial Intelligence. (1998)

11. XSens Motion Technologies: Xsens inertial measurement unit product description and documentations (2006) 